\title{
Atypical Gastric Lymphoid Hyperplasia
}

National Cancer Institute

\section{Source}

National Cancer Institute. Atypical Gastric Lymphoid Hyperplasia. NCI Thesaurus. Code C9472.

An atypical lymphoid hyperplasia involving the gastric mucosa. 